\title{
Evaluation of Haptic and Visual Cues for Repulsive or Attractive Guidance in Nonholonomic Steering Tasks
}

\author{
Roel J. Kuiper, Dennis J. F. Heck, Irene A. Kuling, and David A. Abbink, Senior Member, IEEE
}

\begin{abstract}
Remote control of vehicles is a difficult task for operators. Support systems that present additional task information may assist operators, but their usefulness is expected to depend on several factors such as 1) the nature of conveyed information, 2) what modality it is conveyed through, and 3) the task difficulty. In an exploratory experiment, these three factors were manipulated to quantify their effects on operator behavior. Subjects $(n=15)$ used a haptic manipulator to steer a virtual nonholonomic vehicle through abstract environments, in which obstacles needed to be avoided. Both a simple support conveying near-future predictions of the trajectory of the vehicle and a more elaborate support that continuously suggests the path to be taken were designed (factor 1). These types of information were offered either with visual or haptic cues (factor 2). These four support systems were tested in four different abstracted environments with decreasing amount of allowed variability in realized trajectories (factor 3). The results show improvements for the simple support only when this information was presented visually, but not when offered haptically. For the elaborate support, equally large improvements for both modalities were found. This suggests that the elaborate support is better: additional information is key in improving performance in nonholonomic steering tasks.
\end{abstract}

Index Terms-Guidance forces, haptic feedback, haptic shared control, nonholonomic steering, potential fields, teleoperation, visual support.

\section{INTRODUCTION}

$\mathbf{R}$ EMOTE control, or teleoperation, is much more difficult for operators than direct manipulation due to delays and limited sensory feedback of the task [1], [2]. Visual information from available cameras of the remote location is typically limited (in terms of view angle and depth perception) and makes it difficult to interpret what the actual state of the vehicle or manipulator is [3] and [4]. Vestibular feedback of the remote vehicular movements is inherently absent, unless linked to a motion-based simulator [5]. Moreover, naturally occurring

Manuscript received May 18, 2015; revised October 1, 2015 and February 26 2016; accepted March 15, 2016. Date of publication June 2, 2016; date of current version September 14, 2016. This work was supported by the Dutch Technology Foundation STW (Project 12163), which is part of the Dutch Organization for Scientific Research (NWO) and partly funded by the Ministry of Economic Affairs. This paper was recommended by Associate Editor A. Peer.

R. J. Kuiper and D. A. Abbink are with the Delft Haptics Laboratory, Department of Biomechanical Engineering, Faculty of 3mE, Delft University of Technology, 2628 CD Delft, The Netherlands (e-mail: r.j.kuiper@tudelft.nl, d.a.abbink@tudelft.nl).

D. J. F. Heck is with the Department of Mechanical Engineering, Dynamics and Control Group, Eindhoven University of Technology, 5612 AZ Eindhoven, The Netherlands (e-mail: d.j.f.heck@tue.nl).

I. A. Kuling is with the MOVE Research Institute Amsterdam, Faculty of Human Movement Sciences, Vrije Universiteit Amsterdam, 1081 HV Amsterdam, The Netherlands (e-mail: i.a.kulling@vu.nl).

Color versions of one or more of the figures in this paper are available online at http://ieeexplore.ieee.org.

Digital Object Identifier 10.1109/THMS.2016.2561625 auditory or haptic feedback of the vehicle interaction with the remote environment (e.g., wind gusts, currents, contact) is absent unless reengineered [6].

An alternative to restoring natural sensory feedback is to add artificial task-related feedback. For example, limited visual feedback can be compensated for by using augmented reality such as grid lines to enhance depth perception [3]. Other visual overlays [7]-[9], predictors (flight director, visual guidance for landing planes [8]), or visual feedback can be replaced by virtual reality entirely [4]. Assistive haptic feedback can be presented as artificial guidance forces to the human operator. These guidance forces can be offered as passive guidance forces, also known as virtual fixtures, e.g., a virtual wall to support operators in a peg-in-hole task [10]. They can also be extended to more complex guidance using soft fixtures to create a virtual tunnel [11], [12]. For remote control of aerial vehicles in an unstructured environment, potential fields are commonly used to provide assistive haptic feedback [13]-[15]. This method uses only nearby environment information around the vehicle without involving any task-related strategy and has been applied to subsea robotics [16], needle steering [17], and telemanipulation [19]. The required remote environment information is obtained from sensors on the vehicle.

Another, more comprehensive, approach to support operators with assistive haptic feedback is to use guidance toward an optimal steering input based on a suggested path. The feedback forces, based on a predicted vehicle position, act as a haptic prediction to the suggested path. This concept essentially acts as a second controller and is known as haptic shared control [20], [21]. It has been applied to car driving [20]-[23], subsea robotics [24], teleoperated surgery [25], and nuclear maintenance [26].

Haptic guidance based on potential fields or haptic shared control has either been compared for a one-degree-of-fre $3 \mathrm{dom}$ (DOF) steering task [27], or solely combined for path planning and haptic guidance along an adaptive path [28]. In this study, both haptic guidance types are compared with each other and additionally compared to visual guidance based on similar additional information.

\section{A. Type of Additional Information}

For nonholonomic steering, task-related feedback to the user could come in the form of repulsive or attractive guidance cues. Repulsive cues relate to the likelihood of adverse events, such as collisions with nearby obstacles, whereas attractive guidance cues relate to a desired path throughout the entire environment. To calculate desired steering inputs to either avoid obstacles or follow the suggested path, detailed knowledge of the vehicle kinematics and dynamics is required. In contrast with 
information to avoid obstacles, the suggested path information also conveys strategic information and requires prerequisite knowledge of the task and the environment.

Furthermore, information can be conveyed through different modalities: either visually or haptically. An advantage of using the visual modality is that the information about the position and the required action can be very detailed. On the other hand, the visual channel can be easily overloaded, and other modalities have to be considered. Presenting information through the haptic channel is typically faster, since it enables the operator to respond to the forces through fast reflexes [8], [9], [22]. To compare the types of additional information between modalities, the calculated near-future predicted trajectory can be visualized to the operator by using augmented reality, or felt as an artificial haptic potential field. The same applies to the suggested path, which can both be visualized or reflected as a haptic shared controller.

The usefulness of the support system not only depends on the design of the support, but also on the difficulty of the task. Nonholonomic steering, used for vehicles with front wheel steering, is a common but relatively complex kinematic system to control [29]. The input relation between the steering angle and the resulting turning radius of the vehicle is easily underestimated [17]. When the task environment requires sharp steering angles close to the minimal turning radius of the vehicle, e.g., to avoid obstacles along the way, the operator has limited options to change the current trajectory. The support system should help the operator decide the timing and magnitude of the correct steering inputs with respect to the spatial-temporal constraints of the environment. Depending on the task difficulty, either a local trajectory prediction to avoid obstacles or a suggested path incorporating a strategy to avoid obstacles throughout the entire environment might be more beneficial.

\section{B. Experimental Outline}

In this study, a human factor experiment is conducted to test the effect of different types of information (predicted vehicle trajectory and suggested path, factor 1), presented in different modalities (visually and haptically, factor 2), on the performance of a nonholonomic steering task in environments of varying difficulty (factor 3 ). This is accomplished by using a haptic input device and a virtual abstract vehicle driving through several virtual mazes of varying difficulty. In this experiment, we aimed to compare the most promising ways to present to the operator identical sensor information through either the visual or the haptic channel.

It takes longer for the operator to process and react upon the visual information compared to the haptic information, since in the latter case the haptic guidance only needs to be agreed on. Therefore, it is hypothesized that receiving the information haptically would result in improved performance and reduced control effort compared to receiving the additional information visually (factor 2). Second, it is hypothesized that there is an interaction between the type of additional information (factor 1) and the task difficulty based on different environments (factor 3 ). It is expected that more information is particularly useful when the task difficulty is high. This aspect could be reflected in the interaction by improved performance and reduced effort; there will only be a performance benefit when the additional information of a suggested path is presented in environments with obstructed targets and not in environments with reduced distance between obstacles.

\section{SUPPORT DESIGN}

In this study, four types of support systems were designed and evaluated, each constituting a combination of two factors. The design included either only local environment information or also global task information. Additionally, the support system was either communicated via haptic or visual cues.

During all conditions, the vehicle, obstacles, and target location were shown on the visual display and transparent feedback forces (i.e., vehicle dynamics and contact forces) were reflected on the translational direction of the input device (further described in Section III-B). The haptic support systems were offered as a torque on the planar rotational steering direction of the input device. As a result, the reflected torques are decoupled from the natural forces and assist the operator in steering the vehicle. The following sections describe the different forms of haptic and visual support systems in more detail.

\section{A. Repulsive Haptic Guidance Around Obstacles}

Repulsive haptic guidance is defined as a haptic potential field around the obstacles to assist the subjects in avoiding contact, similar to [14]. Haptic guidance torques are generated based on a predicted position of the slave after a translation of $L_{p}$ with the current orientation input of the master device. When the predicted slave position is within the detection distance $d_{p}$ of the obstacle [see Fig. 1(a)], a virtual repulsive force is applied on a distance $L_{p}$ from the current slave position. This virtual force is proportional, with gain $k_{p}$, to the penetration depth $d_{p}-d_{s}$. Furthermore, the repulsive forces are only reflected to the subjects when the slave tends to move toward an obstacle. This is done by activating the repulsive fields only when the obstacle is within an angle $\alpha$ of the orientation of the slave. Finally, in order to make a fair comparison with the application of attractive guidance, an attractor is designed around the target area. Apart from a negative stiffness gain $k_{s}$, the design of the attractor is similar to the design of the repulsive haptic guidance. For each obstacle $i$, the resulting repulsive guidance torque is implemented as

$$
\tau_{\mathrm{HR}, i}=\left\{\begin{array}{cc}
k_{p} L_{p}\left(d_{p}-d_{\mathrm{si}}\right), & \text { if } d_{\mathrm{si}} \leq d_{p} \text { and }\left|\theta_{i}\right| \leq \alpha \\
0, & \text { else. }
\end{array}\right.
$$

The guidance torque presented to the subjects is the sum of the guidance torques $\tau_{\mathrm{HR}, i}$ for all obstacles, i.e.,

$$
\tau_{\mathrm{HR}}=\sum_{i=1}^{n} \tau_{\mathrm{HR}, i}
$$

with $n=5$ the number of obstacles and attractors. The parameters were tuned to provide an informative repulsive torque that can be overruled by the subjects. The obtained values 


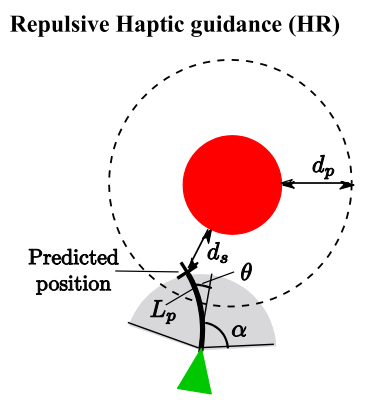

(a)

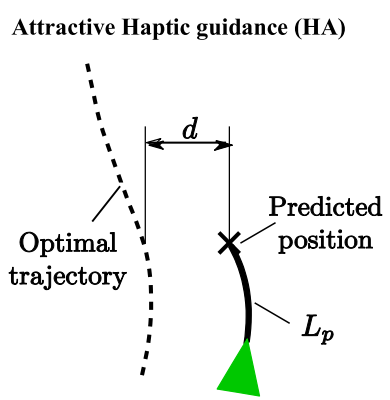

(b)

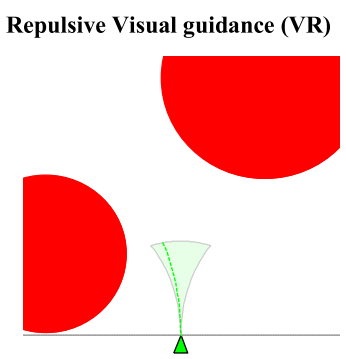

(c)

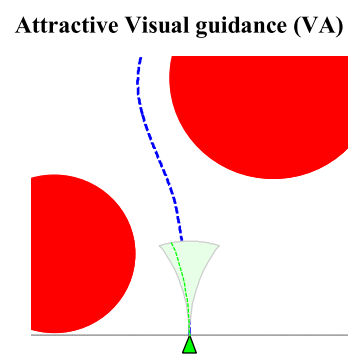

(d)

Fig. 1. Representation of the underlying information required to generate the haptic support, in their simple realizations and their more elaborate realizations, and how this was displayed for their visual equivalent. (a) Repulsive haptic guidance (HR) around an obstacle, based on the predicted slave position. (b) Attractive haptic guidance (HA) to a suggested path also based on the predicted slave position. (c) Example of repulsive visual guidance (VR) displaying the predicted slave position visually in green. (d) Example of attractive visual guidance (VA), displaying the entire suggested path in blue throughout the environment in combination with the predicted slave position.

are $L_{p}=d_{s}=0.01 \mathrm{~m}, \alpha=90^{\circ}$, and $k_{p}=6 \mathrm{~N} / \mathrm{m}$ for the repulsors and $k_{p}=-6 \mathrm{~N} / \mathrm{m}$ for the attractor of the target.

\section{B. Attractive Haptic Guidance to a Suggested Path}

Attractive haptic guidance torques guide the operator toward a predefined suggested path from the starting position to the target location, similar to [22]. The designed suggested paths avoid all obstacles with a minimal distance, equal to the distance $d_{p}$ used for repulsive feedback (see Section II-A), and consist of radii equal or larger than the minimal turning radius of the vehicle. The blue dashed line in Fig. 1(c) shows an example of such a suggested path through an environment.

Comparable with the repulsive guidance, torques are computed from a virtual guidance force acting on an arm $L_{p}$ from the current slave position. This virtual guidance force is proportional, with gain $k$, to the horizontal distance $d$ between the suggested path and a predicted position of the slave [see Fig. 1(b)]. The sign of the virtual guidance force is related to the relative horizontal position of the suggested path with respect to the predicted slave position and results in a guidance torque towards the suggested path. The predicted position of the slave is updated online and obtained by computing the slave position after a translation of $L_{p}$ with the current orientation input of the master device. By rotating the master device, the predicted position of the slave is affected, resulting in a change in the distance $d$. As a result, the subjects feel a torsional stiffness on the master device. The attractive guidance torque is implemented as

$$
\tau_{\mathrm{HA}}=k L_{p} d
$$

The distance $L_{p}$ and stiffness $k$ were tuned to provide an informative assistive torque that can be overruled by the subjects. The obtained values are $L_{p}=0.01 \mathrm{~m}$ and $k=5 \mathrm{~N} / \mathrm{m}$.

\section{Visual Equivalent Support System}

The haptic guidance is either based on information of the suggested path (attractive guidance) or information to prevent collisions with the obstacles (repulsive guidance). In order to investigate if the transmitted type of additional information or the reflection via the haptic channel results in improved performance for this task, this information is also visually represented.

In the visual equivalent of the repulsive haptic guidance, subjects received visual information regarding the predicted slave position. The predicted slave position is indicated by a green dashed line starting from the front of the slave [see Fig. 1(c)]. This line informs the subjects where the slave will be when the master is translated $L_{p}=1 \mathrm{~cm}$ with the current orientation. The green area reflects the range of possible slave steering angles, taking into account the maximum steering radius.

In the visual version of the attractive guidance, subjects additionally received information regarding the suggested path, indicated with a blue dashed line from start to target. Both the predicted slave position and the suggested path are visualized, as shown in Fig. 1(d).

\section{EXPERIMENTAL METHODS}

\section{A. Subjects}

Fifteen subjects, ten males and five females, with an average age of 26.7 years and 3.8 year standard deviation, volunteered for the experiment. All subjects had no experience with teleoperation and were naive about the experiment. All subjects gave their written informed consent prior to the experiment. The setup and experiments were approved by the local ethics committee of the Delft University of Technology.

\section{B. Apparatus}

The experiments were performed using a three DOF planar parallel master device (as depicted in Fig. 2) [30] and a virtual slave, running on a Mathworks xPC Target real-time operating system at $1 \mathrm{kHz}$ with an estimated time delay between master and slave of $1.5 \mathrm{~ms}$.

Subjects were holding a knob at the center of rotational input of the master device and controlling the horizontal translation and rotation. The forward translation of the master device was coupled to the radial translation of the virtual slave. To make the coupling to the slave tangible, a translational damping field of $50 \mathrm{~N} \cdot \mathrm{s} / \mathrm{m}$ was applied. The rotation of the master device was coupled to the steering of the virtual slave. The lateral translation 


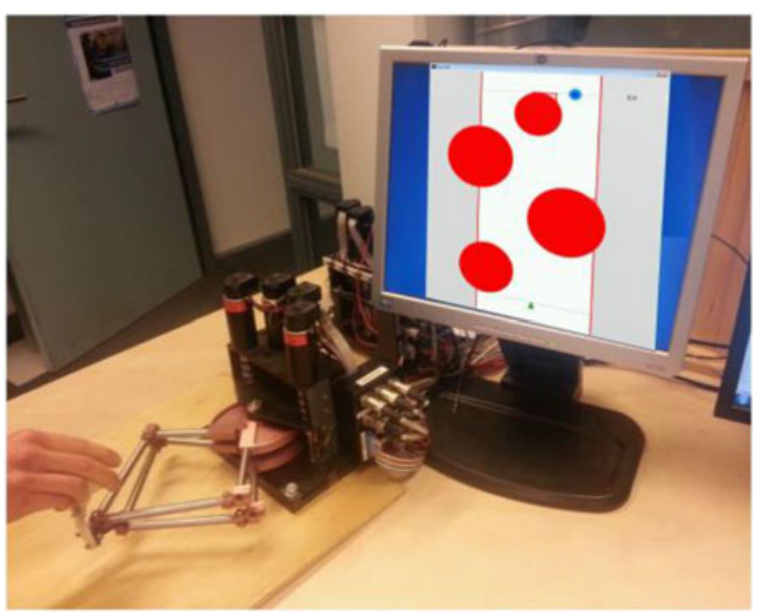

Fig. 2. Experimental setup showing an operator holding the planar haptic master device and the display showing the virtual slave and environment.

of the master device was constrained with a stiffness of $400 \mathrm{~N} / \mathrm{m}$ and not used for controlling the virtual slave.

The dynamics of the slave are represented by a planar secondorder bicycle model of two DOFs (orientation and radial translation) [29]. The virtual vehicle has a theoretical wheelbase of $0.05 \mathrm{~m}$, with maximum steering angle of the front axle of $60^{\circ}$. It is represented as a dimensionless point, based on the rear wheel position represented by a green triangle as shown in Fig. 1. The orientation of the vehicle is only affected when both the steering angle and forward or backward translation of the vehicle are controlled. Both forward and backward motion are allowed for master and slave, such that reversing of the slave is enabled. When reversing, the bicycle model is used with negative translational vehicle velocity such that the direction of steering is reversed. The translation of the slave is restricted when it collides with an obstacle. Subjects must reverse the slave direction in order to continue the trial.

\section{Virtual Environment}

The four virtual environments in which the slave operates measure 5 by $10 \mathrm{~cm}$, as shown in Fig. 3. The slave starts at the horizontal line at $x=0 \mathrm{~m}$ and the target is located on a horizontal line at $x=0.1 \mathrm{~m}$. The location of the target area on this horizontal line is indicated by three coincided circles (of 1-, 2-, and 3-cm diameter) of different shades of blue. The obstacles are indicated by the red circles and a green triangle represents the slave. The whole virtual environment including all obstacles and the target was always shown visually to the subjects.

Two factors of spatiotemporal constraints were varied in the experiment, namely the space between obstacles and the difficulty of reaching the target by partly blocking the approach. The distance between the edge of the first and second obstacles $\left(d_{1}\right)$ was varied between 9 and $15 \mathrm{~mm}$, the distance between the second and third obstacle $\left(d_{2}\right)$ was varied between 10 and $15 \mathrm{~mm}$, and the horizontal distance between the last object and the target $\left(d_{\mathrm{tg}}\right)$ ranged between 5 and $-1 \mathrm{~mm}$. The four different environments, shown in Fig. 3, are defined as follows.
EE Easy to avoid obstacles $\left(d_{1}=15, d_{2}=15\right)$ and Easy to reach the target location $\left(d_{\mathrm{tg}}=5\right)$;

DoEt difficult to avoid obstacles $\left(d_{1}=9, d_{2}=10\right)$, but easy to reach the target location $\left(d_{\mathrm{tg}}=5\right)$;

EoDt easy to avoid obstacles $\left(d_{1}=15, d_{2}=15\right)$, but difficult to reach the target location $\left(d_{\mathrm{tg}}=-1\right)$;

$D D \quad$ difficult to avoid obstacles $\left(d_{1}=9, d_{2}=10\right)$ and difficult to reach the target location $\left(d_{\mathrm{tg}}=-1\right)$.

\section{Experiment Design}

During the experiments, the following five experimental conditions were presented in separate blocks to assist the subjects in completing the task. The colors used in the figures of Section IV are stated between the parentheses.

NO no haptic or visual guidance. This condition forms the benchmark (blue);

$V R$ visual repulsive information about the predicted slave position (red);

$V A$ visual attractive information about both the predicted slave position and suggested path (orange);

$H R$ haptic repulsive guidance, resulting from the predicted slave position and the objects (dark green);

$H A$ haptic attractive guidance resulting from the predicted slave position and the suggested path (light green).

During the experiments, each block consisted of eight trials with one of the five experimental conditions listed above (no support or one of the four support systems). In these eight trials, the four different environments were presented twice, once as shown in Fig. 3 and once a version mirrored about the vertical axis. Environmental information regarding the objects and target is visually shown during all experimental conditions, as described in the previous section. For all experimental conditions, an additional catch-trial of the difficult environment (DD) was used at the end of each block to investigate the dependency of the subjects on the support system. Therefore in total, each subject was presented with 45 trials.

The order of the five experimental conditions and the order of the presented environments within one condition block were both counterbalanced using a balanced Latin square design [31].

\section{E. Procedure}

Each subject was asked to take place in front of a planar threeDOF parallel master device, as depicted in Fig. 2. The display behind the master device showed several red circular obstacles in the virtual environment. Contact of the virtual slave with these obstacles had to be avoided. The subjects were asked to move the slave as close as possible to the center of the target, but over the finish line, and as fast as possible, but without colliding with any of the obstacles. When the subject crossed the target line, the trial ended. Throughout the entire trial, the travelled trajectory of the slave was shown with a thin green line.

Prior to each experimental condition, the subjects were presented with several trials in a training environment to learn to operate the system and get familiar with the applied form of support system. All subjects were trained up to a minimal required level of performance, consisting of three sequential 


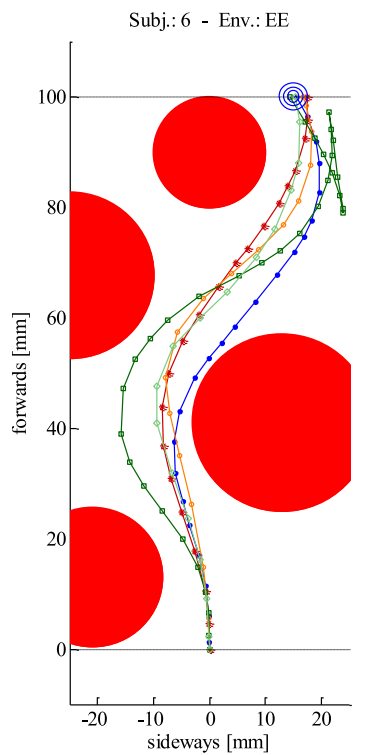

(a)

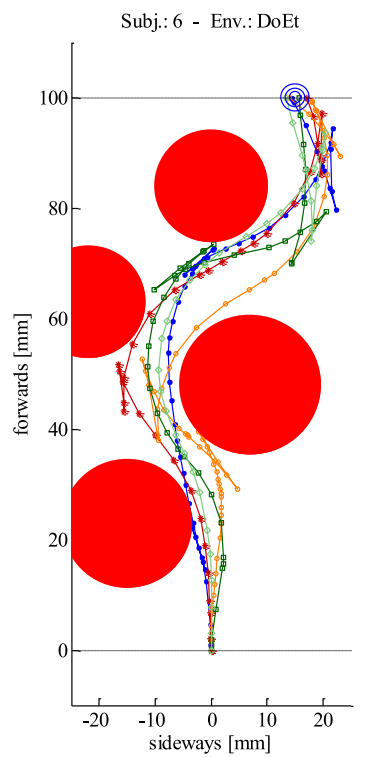

(b)

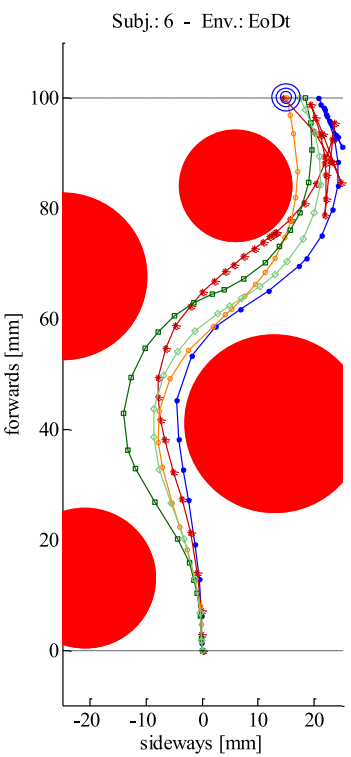

(c)

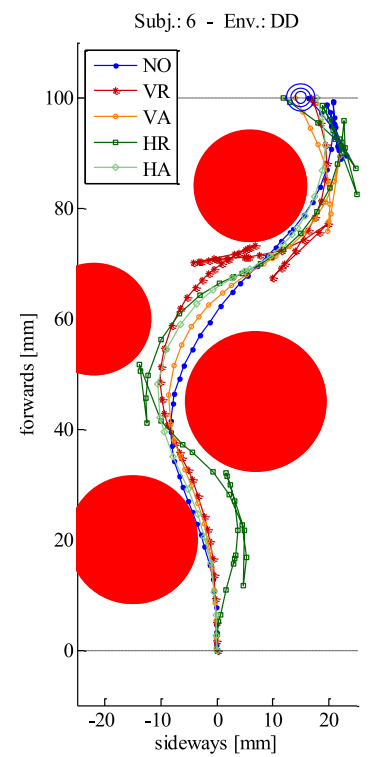

(d)

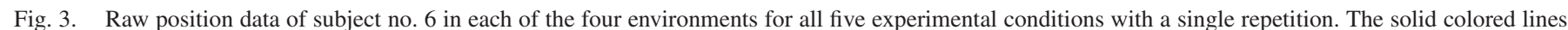

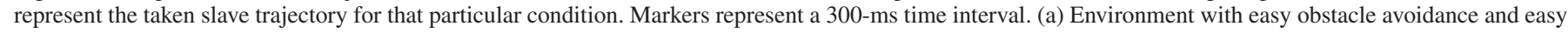

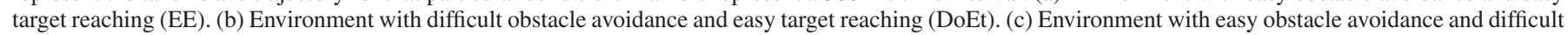
target reaching (EoDt). (d) Environment with difficult obstacle avoidance and difficult target reaching (DD).

successful training trials in two different training environments, having no obstacle collisions or slave retractions, and a completion time of under ten seconds for each trial. The two training environments consisted of either a single obstacle for familiarization or two obstacles for strategic training of a correct task execution.

\section{F. Measured Variables and Metrics}

Analyzing different support systems can be done on many different aspects and metrics. To get an overview of different effects of the environment and form of support, a wide variety of metrics is selected. These metrics are roughly categorized in two groups, namely in general performance and safety metrics, and in effort metrics.

1) General Performance and Safety Metrics: The general performance metrics are task- and goal-related measures on how well the task was executed. The safety metrics reflect measures on the risks taken during the executed tasks. Four different metrics are considered for performance (task completion time, targeting accuracy, number of slave retractions, and mean duration of retractions) and three for safety (number of collisions, minimum time to obstacle collision, and distance to obstacles) to compare the five experimental conditions in four environments.

a) Task completion time: The time it took the subject to complete the task, from the starting line to the horizontal target line.

b) Targeting accuracy: The horizontal distance between the tip of the slave and the center of the target location when the slave crosses the horizontal target line. The targeting error reflects the accuracy at the end of each trial. c) Number of slave retractions: The amount of times the slave transversal direction (back and forward) was changed, i.e., the number of sign changes of the transversal velocity of the slave. This indicates the number of wrong trajectories taken during a trial and is therefore a measure of performance of the correctness of the taken trajectory. The number of retractions were calculated using a 10-Hz Butterworth filter and a deadband threshold of $1.0 \mathrm{~mm} / \mathrm{s}$ on the transversal velocity of the slave. A typical slave retraction is shown in Fig. 3(a) in the top right close to the target for the HR condition (dark green dotted line).

d) Total duration of retractions: The total time it took for all slave retractions during a single trial.

e) Number of collisions: The total number of collisions with obstacles, based on the tip of the slave and the edges of the obstacles.

f) Minimum time to obstacle collision: The minimum time until colliding with an obstacle, considering the current vehicle state (i.e., velocity and orientation) and the current control inputs at the master device. This metric was calculated similarly to the trigonometric computation of time to lane crossing of [32], calculated for each measured frame and for each obstacle, to obtain the minimum of all four obstacles over an entire trial. Note that if there is any collision with an obstacle in a trial, this metric equals zero.

g) Distance to obstacles: The absolute distance to the different obstacles, regardless of the direction of motion, master orientation or velocity, is measured during the trial. This metric is defined as the minimum value of the absolute distance to the obstacles. A collision results in a zero distance.

2) Effort Metrics: The effort metrics are based on objective and subjective measures to reflect the control effort of the operator during the tasks. Two objective (master and slave 


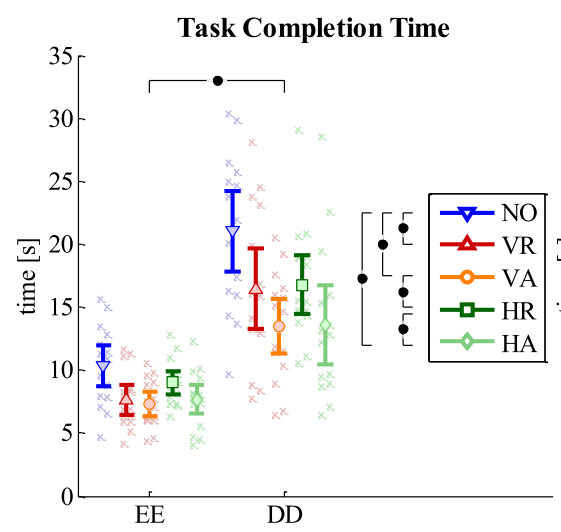

(a)

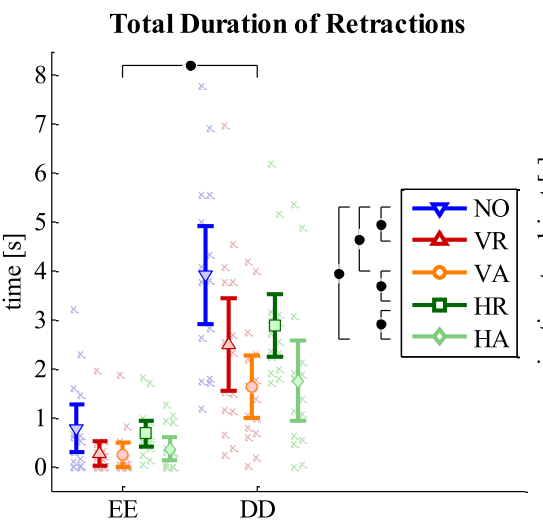

(b)

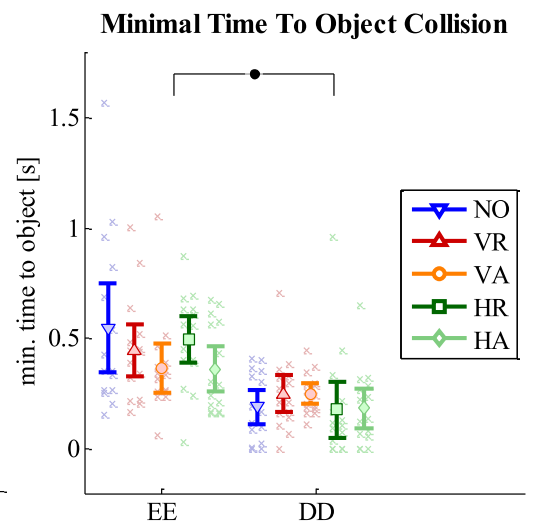

(c)

Fig. 4. Performance and safety results of each experimental condition, for clarity only shown in the two most different environments EE and DD. (a) Task completion time results. (b) Number of slave retractions results. (c) Minimal time to object collision results. The dots and x's represent the average individual result of two trials per subject $(N=15)$, the circles represent the group mean, and the error bars the $95 \%$ confidence interval of the mean. The horizontal and vertical bars indicate a significant difference over the factor environment or support, where "•" denote the significance of $p<0.05$.

TABLE I

STATISTICS OF PERFORMANCE AND SAFETY RESULTS

\begin{tabular}{|c|c|c|c|c|c|}
\hline \multirow[t]{2}{*}{ Metric } & \multicolumn{3}{|c|}{ RM ANOVA } & \multicolumn{2}{|c|}{ Post hoc comparisons } \\
\hline & ENVIRONMENT & Support & Interaction & Environment & Support \\
\hline $\begin{array}{l}\text { Task completion } \\
\text { time }\end{array}$ & $\begin{array}{l}F_{1.8,24.9}= \\
\quad 72.5 \\
p<0.001\end{array}$ & $\begin{array}{l}F_{4,56}=15.7 \\
\quad p<0.001\end{array}$ & $\begin{array}{l}F_{5.1,71.7}= \\
2.1, p=0.07\end{array}$ & $\begin{array}{l}\text { All comparisons } p<0.001 \text { except DoEt and } \\
\text { DD } p=0.15\end{array}$ & $\begin{array}{l}\text { No and VR, VA, HA } p=0.010, p<0.001 \\
\text { and } p<0.001 \text { respectively } \\
\text { HR and VA, HA } p=0.002, p=0.003 \\
\text { respectively }\end{array}$ \\
\hline Target accuracy & $\begin{array}{l}F_{3,42}=2.8 \\
p=0.054\end{array}$ & $\begin{array}{c}F_{4,56}=6.8 \\
p<0.001\end{array}$ & $\begin{array}{c}F_{5.3,74.2}= \\
2.6, p=0.027\end{array}$ & - & $\begin{array}{l}\text { No and VA } p=0.043 \\
\text { HR and VA } p=0.004 \\
\text { No and VR, VR and HR, VA and HA, all } \\
p=0.08\end{array}$ \\
\hline $\begin{array}{l}\text { No. slave } \\
\text { retractions }\end{array}$ & $\begin{array}{l}F_{1.9,26.3}= \\
\quad 52.4 \\
p<0.001\end{array}$ & $\begin{array}{c}F_{2.1,29.9}= \\
8.8, p=0.001\end{array}$ & $\begin{array}{l}F_{12,168}=1.5 \\
\quad p=0.12\end{array}$ & $\begin{array}{l}\text { All comparisons } p<0.001 \text { except for DoEt } \\
\text { and DD } p=0.62\end{array}$ & $\begin{array}{l}\text { No and VA, HA } p=0.018, p=0.062 \\
\text { respectively } \\
\text { HR and VR, VA, HA } p=0.001, p<0.001 \text {, } \\
\text { and } p<0.008 \text { respectively }\end{array}$ \\
\hline $\begin{array}{l}\text { Total duration of } \\
\text { retractions }\end{array}$ & $\begin{array}{l}F_{1.9,27.2}= \\
\quad 51.5 \\
p<0.001\end{array}$ & $\begin{array}{l}F_{2.3,32.2}= \\
\quad 10.8 \\
p<0.001\end{array}$ & $\begin{array}{c}F_{5.1,1.6}= \\
2.2, p=0.065\end{array}$ & $\begin{array}{l}\mathrm{EE} \text { and DoEt, EoDt, DD all } p<0.001 \text {, EoDt } \\
\text { and DoEt, DD, all } p<0.001\end{array}$ & $\begin{array}{l}\text { No and VR, VA, HA } \\
p=0.024, p<0.001, p=0.004 \\
\text { respectively } \\
\text { HR and VA, HA } p<0.001, p=0.032 \\
\text { respectively }\end{array}$ \\
\hline $\begin{array}{l}\text { Min. time to } \\
\text { obstacle coll. }\end{array}$ & $\begin{array}{c}F_{3,42}=30.6 \\
p<0.001\end{array}$ & $\begin{array}{c}F_{4,56}=1.9 \\
p=0.13\end{array}$ & $\begin{array}{l}F_{4.9,68.3}= \\
1.8 p=0.13\end{array}$ & $\begin{array}{l}\text { EE and DoEt, EoDt, DD } p<0.001 \\
\text { DoEt and EoDt } p=0.005\end{array}$ & - \\
\hline No. of collisions & $\begin{array}{c}F_{1.9}, 27.7= \\
15.5 \\
p<0.001\end{array}$ & $\begin{array}{l}F_{2.2,30.8}= \\
3.8, p=0.029\end{array}$ & $\begin{array}{c}F_{2.4,33.5}= \\
0.8, p=0.47\end{array}$ & $\begin{array}{l}\mathrm{EE} \text { and } \mathrm{DoEt}, \mathrm{EE} \text { and } \mathrm{DD}, \\
p<0.001, p=0.006 \text { respectively } \\
\text { EoDt and } \mathrm{DD}, \mathrm{EoDt} \text { and DoEt, } \\
p=0.006, p=0.001 \text { respectively }\end{array}$ & No and VA $p=0.043$ \\
\hline $\begin{array}{l}\text { Distance to } \\
\text { obstacles }\end{array}$ & $\begin{array}{l}F_{3,42}=472 \\
\quad p<0.001\end{array}$ & $\begin{array}{l}F_{4,56}=2.0 \\
\quad p=0.11\end{array}$ & $\begin{array}{c}F_{12,168}=2.0 \\
p<0.030\end{array}$ & $\begin{array}{l}\text { All comparisons } p<0.001 \\
\text { except for EE and } \operatorname{EoDt}(p=0.0 .3) \text { and DoEt } \\
\text { and DD } p=1.0\end{array}$ & - \\
\hline
\end{tabular}

Using a two-way repeated measures ANOVA on environment and support, and the interaction between the two. The post hoc analysis shows the differences per condition or environment type if RM ANOVA showed differences.

reversals) and four subjective metrics (TLX, comfort, effort, and preference rating) are selected to compare five experimental conditions in four environments.

a) Reversals master: As used in [26], the number of steering corrections was used as a measure for control effort because it requires a conscious decision to change the sign of steering direction. This measure is defined as the number of steering reversals on the master input, i.e. the number of sign changes of the rotational velocity of the master, using a 10-Hz Butterworth filter. A $1.0^{\circ}$ threshold was used as deadband on the filtered signal to filter out unintentional oscillations of the subjects.

b) Reversals slave: The reversals of the slave were computed in a similar way as the reversals of the master. The slave only rotates when the subjects combine a change in rotation of 
the master device with a translation. Therefore, the reversals of the slave represent a more long-term change in steering angle, while the reversals of the master also show the more short-term changes of the steering and repositioning of the master device to make steering easier. A slave rotational reversal differs from a slave retraction because it represents the change of heading, not the back and forward transversal direction change.

c) Subjective measures: The NASA Task Load Index (NASA-TLX) was used to measure workload [33]. Furthermore, the subjects were asked to rank the five experimental conditions for comfort, effort, and preference.

\section{G. Data Analysis}

For each subject, form of support system, and environment, the metrics are computed per trial and averaged over two repetitions. Per metric, the means are compared between the forms of support and environment, and possible interactions with a $4 \times$ 5 (environment $\times$ support) repeated measures analysis of variance (RM-ANOVA). A Greenhouse-Geisser correction was applied when sphericity was violated. For significant main effects $(p<0.05)$, post hoc comparisons with Bonferroni correction for multiple comparisons were done to get more insight to the origins of the effects.

\section{RESULTS}

\section{A. Position Data}

The raw position data of a representative subject are shown in Fig. 3 for each of the four environments, including all five conditions with two repetitions in every environment. The data show smooth curvatures for the easy environment (EE) with different types of curves between the obstacles, clearly showing the available freedom for different strategies to reach the target. The same occurs at the first part of the environment EoDt, but the lines converge and more retractions can be seen near the end. For the DoEt and DD environments, retractions are visible throughout the whole environment.

It can also be seen in Fig. 3 that the HR condition (dark green) shows a different trajectory compared to the other conditions, resulting for the difficult environment (DD) in collisions in the beginning of the trial. It is a clear indication of the drawback of this local feedback, which does not include information regarding the next obstacle.

\section{B. Performance and Safety Results}

The results on all task performance metrics, as explained in Section III-F, are described here. The analyses are done on all environments and all support systems. For clarity, the results presented in Fig. 4 only show the results of the two most different environments EE and DD. Furthermore, the text only describes the main findings, but all statistical details of all RM ANOVA's can be found in TABLE I. The specific differences between the results in the NO support and the other experimental conditions for all environments can be seen in TABLE III.
1) Task Completion Time: The mean completion time over all subjects, trials, and conditions was $12.8 \mathrm{~s}$. The completion times in the NO condition are significantly larger (statistical details can be found in Table I) than in VR, VA, and HA, and the HR condition took significantly longer than VA and HA [see Fig. 4(a)]. This suggests that additional information about both the vehicle kinematics and the suggested path decrease task completion time. Moreover, a significant influence of the environment is observed; both a more narrow environment and a more difficult to reach target influence the completion time significantly. However, the narrow environments had the most effect, as can be seen in TABLE III.

2) Targeting Accuracy: The type of environment did not had a significant effect on the target accuracy. The support system did have a significant effect and there was a significant interaction between the environment and support (see Table I). Post hoc comparisons on support show that the errors in the $x$-directions are $(p<0.05$, or have a tendency $(p=0.08)$ to be $)$ smaller in the visual conditions compared with the NO and HR condition. The substantial difference is very low (ranging for the mean NO condition from 1.5 to $2.2 \mathrm{~mm}$; see Table III), compared with the target size of $3 \mathrm{~mm}$ (see Section III-C).

3) Number of Slave Retractions: Both the environment and support had a significant effect on the number of slave retractions (see Table I). There are less retractions for the visual conditions and the HA, and more for the NO and the HR conditions, which suggests that more additional information leads to less slave retractions. The number of slave retractions is significantly lower for environments EE and EoDt compared with the other environments (see Table I). This shows that the number of slave retractions increases with both the narrowness of the environment and the difficulty to reach the target. The combination of the two does not increase the amount of slave retractions further.

4) Total Duration of Retractions: A significant effect for both the environment and support was found for the total duration of retractions (see Table I). Both visual supports and HA have significant less duration of the retractions than the NO condition [see Fig. 4(b)]. The duration of retractions in the easy environment (EE) was significantly shorter than in all other environments, followed by those in the EoDt, indicating that increasing task difficulty results in longer retractions.

5) Number of Obstacle Collision: The number of obstacle collisions depends strongly on the narrowness of the environment and thereby the difficulty to avoid the obstacles. Environments with large distances between obstacles (EE and EoDt) had significantly less obstacle collisions compared to narrow environments (DoEt and DD) (see Table I).

6) Minimum Time to Obstacle Collision: For the minimum time to obstacle collision, only a significant effect for the environment was found (see Table I). In the easy environment (EE), the time to obstacle collision was larger than in all other environments [see Fig. 4(c)].

7) Distance to Obstacles: The distance to obstacles depends significantly on the environment. Larger distances between the obstacles result in larger distances between the slave and the obstacles (see Table I). 


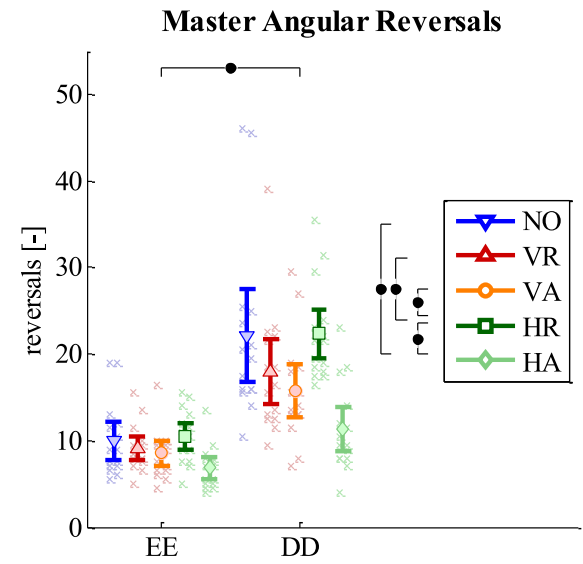

(a)

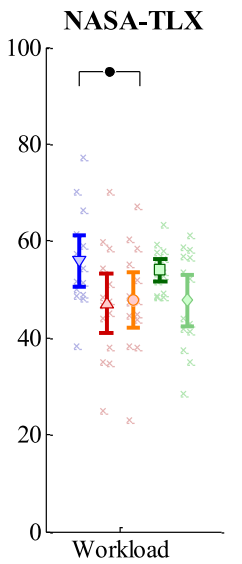

(b)

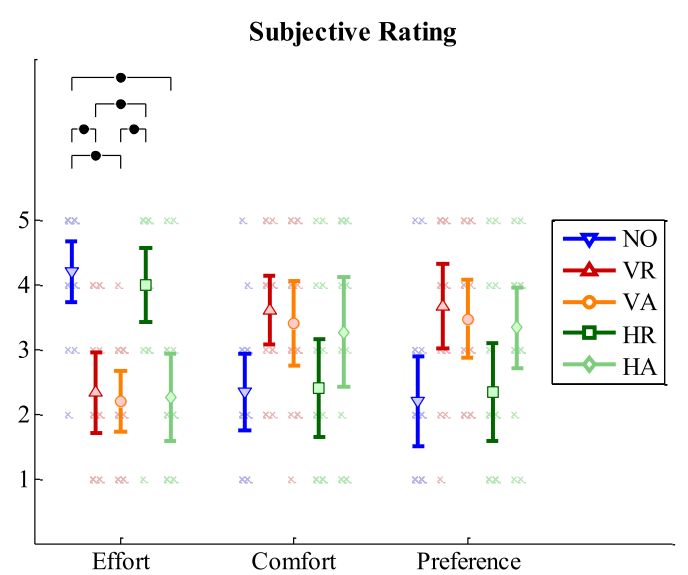

(c)

Fig. 5. Effort results of each experimental condition, for clarity only shown in the two most different environments EE and DD. (a) Number of master reversal results. (b) NASA TLX workload results. (c) Subjective ratings of each experimental condition over all environments. General figure representations of metrics are described in the caption of Fig. 4.

TABLE II

STATISTICS OF EFFORT RESULTS

\begin{tabular}{|c|c|c|c|c|c|}
\hline \multirow[t]{2}{*}{ Metric } & \multicolumn{3}{|c|}{ RM ANOVA } & \multicolumn{2}{|c|}{ Post hoc comparisons } \\
\hline & ENVIRONMENT & Support & Interaction & Environment & Support \\
\hline No. master reversals & $\begin{array}{l}F_{2.2,31.3}=48.5 \\
\quad p<0.001\end{array}$ & $\begin{array}{c}F_{4,56}=13.6 \\
p<0.001\end{array}$ & $\begin{array}{l}F_{3.9,55.1}=2.0 \\
\quad p=0.11\end{array}$ & $\begin{array}{l}\text { All comparisons } p<0.001 \text { except } \\
\text { for DoEt and DD } p=1.0\end{array}$ & $\begin{array}{l}\mathrm{NO} \text { and HA } p=0.006 \mathrm{HR} \text { and VR, } \\
\mathrm{VA}, \mathrm{HA} \\
p=0.021, p<0.001, p<0.001 \\
\text { respectively }\end{array}$ \\
\hline No. slave reversals & $\begin{array}{l}F_{3,42}=53.4 \\
\quad p<0.001\end{array}$ & $\begin{array}{l}F_{2.0,27.5}=9.9 \\
\quad p=0.001\end{array}$ & $\begin{array}{l}F_{3.9,54.5}=2.1 \\
\quad p=0.09\end{array}$ & $\begin{array}{l}\text { All comparisons } p<0.001 \text { except } \\
\text { for DoEt and DD } p=1.0\end{array}$ & $\begin{array}{l}\mathrm{NO} \text { and VR, VA, HA } \\
p=0.044, p<0.001, p=0.050 \\
\text { respectively } \\
\text { HR and VR, VA, HA } \\
p=0.001, p<0.001 \text { and } \\
p=0.039 \text { respectively }\end{array}$ \\
\hline NASA-TLX & & $\begin{array}{l}F_{4,52}=4.7 \\
p=0.003\end{array}$ & & & $\begin{array}{l}\text { No and VA, HA } \\
p=0.043, p=0.07 \text { respectively }\end{array}$ \\
\hline \multirow[t]{2}{*}{ Effort rating } & & $\begin{array}{l}F_{4,56}=9.6 \\
p<0.001\end{array}$ & & & $\begin{array}{l}\text { No and VR, VA, HA } \\
p=0.002, p=0.004, p=0.011 \\
\text { respectively }\end{array}$ \\
\hline & & & & & $\begin{array}{l}\mathrm{HR} \text { and } \mathrm{VR}, \mathrm{VA} \\
p=0.046, p=0.003 \text { respectively }\end{array}$ \\
\hline Comfort rating & & $\begin{array}{c}F_{4,56}=2.3 \\
p=.073\end{array}$ & & & - \\
\hline Preference rating & & $\begin{array}{l}F_{4,56}=3.2 \\
p=0.020\end{array}$ & & & - \\
\hline
\end{tabular}

Using a two-way repeated measures ANOVA on environment and support, and the interaction between the two. The post hoc analysis shows the differences per condition or environment type if RM ANOVA showed differences.

\section{Effort Results}

The results for the safety and effort metrics (see Section III-F) are described here. Again for clarity, Fig. 5(a) only presents the results of environments EE and DD. All specific differences can be seen in Table III. Statistical details of all RM ANOVAs can be found in Table II.

1) Slave and Master Angular Reversals: Angular reversals, relating to steering corrections, are analyzed for both the master and the slave. For the slave, there are more reversals in the $\mathrm{NO}$ and HR conditions compared to the other conditions, while for the reversals of the master a benefit of HA over the visual conditions can be seen [see Table III and Fig. 5(a)]. HA has less master reversals than in the NO condition, while the amount of master reversals in the visual conditions do not significantly differ from the NO condition.

Both the master and slave reversals were significantly lower for $\mathrm{EE}$ and EoDt compared to the other environments. This shows that the number of reversals increases with both the narrowness of the environment and the difficulty to reach to target. The combination of the two does not increase the amount of master reversals further. This is in line with the obtained results for the number of slave retractions.

2) NASA-TLX and Subjective Ratings: In all subjective measures, a clear trend is observed in favor of additional information, except when offered HR support. The workload measured with the NASA-TLX [see Fig. 5(b)], and effort ranking [see 
Fig. 5(c)], is higher for the NO and HR conditions, while they score lower on Comfort and Preference.

\section{Catch Trials}

The results of the catch trials at the end of each condition in the difficult environment DD are shown in Fig. 6. The normal conditions are depicted in color and the catch trials in gray. The normal conditions are similar as shown before and consist of individual means of two repetitions, whereas the catch trials had only one repetition per condition. The results show no difference between the trials of each condition in the normal situation and the catch trials. Only for the HA condition is an increasing trend observed for the catch trial for completion time, retractions, and collisions due to several extreme values.

\section{DISCUSSION}

\section{A. Impact of Two Support Designs on Task Execution}

Most performance and control effort metrics show significant differences for the two support systems (factor 1) for the hypothesized effect that feedback of the predicted vehicle kinematics and the suggested path information is helpful in difficult environments. For example, task completion time significantly improves for all support systems (except for repulsive haptic guidance) compared to pure manual control. Similar results for reduction of task completion times were reported by Boessenkool, Abbink, Heemskerk, van der Helm, and Wildenbeest [26] for haptic guidance along a suggested path. Although not tested extensively, the relative improvements suggest a very consistent pattern for all environments over all metrics. For the task completion time, the averaged relative improvements were $20 \%, 27 \%, 8 \%$, and $28 \%$ for VR, VA, HR, and HA, respectively. This suggests that for all environments there is consistently more improvement when additional information of the suggested path is given compared to only the predicted vehicle trajectory.

\section{B. Impact of Two Modalities on Task Execution}

When the path information is reflected haptically, no difference in performance is found compared to visual support (factor 2). This is in contrast to what Stigter et al. reported in [8], where improvements for haptics over visual support for a flight director for more accurate path following reduced the control effort. Wildenbeest, Kuiper, van der Helm, and Abbink [34] also reported that haptic feedback makes the system dynamics more tangible. For the conducted experiment, the visual operator load was low, therefore enabling the subjects to process the visual cues equally to the haptic cues. In real applications, the visual operator load is typically much higher due to multiple secondary tasks, resulting in larger benefits for haptic cues.

Control effort metrics in this experiment (in terms of master angular reversals) seem to improve the most when suggested path information is given haptically. This is similar to the findings of Griffith and Gillespie [23], who reported an increase in secondary task performance (thus reduced mental workload) during car steering with haptic assistance including suggested path information. Mulder, Abbink, and Boer [35] also found for reduced reversal rates when applying guidance forces including a suggested path for car driving. Boessenkool, Abbink, Heemskerk, van der Helm, and Wildenbeest [26] found similar results for a teleoperation task, in addition to similar TLX results.

In this experiment, only additional haptic cues applied in the form of repulsive haptic guidance resulted in no significant improvements compared to the baseline without support (in terms of task completion time, slave retractions, master angular reversals). Therefore, it seems that the repulsive haptic guidance was not supportive for the given tasks. This result differs from the artificial force field implementation of Lam, Mulder, and Ren [13], who reported that the ability of collision avoidance for varying artificial force fields results in improved task performance. The method for obstacle avoidance of Khatib [36] for full automation of a robotic arm also shows positive results for a time-varying artificial force field around obstacles. The raw time traces shown in Fig. 3 indicate a degrading effect of the repulsive haptic guidance, since it always steers away from obstacles, even when the limited vehicle steering angle requires cutting corners at several points. Providing this information only visually possibly enabled the subjects to extrapolate this information to longer predictions and, therefore, correct the steering input in time.

\section{Impact of Four Environments on Task Execution}

The experimental results showed improvements (in terms of task completion time, number of retractions, number of collisions, and master angular reversal rate) in all four environments of varying spatiotemporal constraints (factor 3 ). The results suggest that the largest improvements were found when more critical constraints (for obstacle avoidance and target reachability) were present in the environment. This indicates that support systems are more effective when the task becomes more difficult.

The designed environments strongly influenced the task execution behavior of the subjects. The experimental results show significant differences (for all performance and objective control effort metrics) for the environments of varying spatiotemporal constraints. Therefore, the absolute metric results show differences for most environment variations. This difference is also visible in the relative improvements in task completion time (see Table III) compared with manual control. For example, most relative metrics show a 5-10\% improvement in the difficult environment. Moreover, the absolute averaged completion time doubles in the difficult environment $(21.0 \mathrm{~s})$ compared to the easy environment (10.4 s). Since the relative improvements remain approximately equal, this still means that the absolute effect of the support systems is doubled. The difficult environments took longer to complete due to their increased difficulty, such that all the support systems are effective in improving the performance.

No difference was found for the hypothesized effect that task criticality influences the required type of additional information. No specific support system improves the task more in a specific 

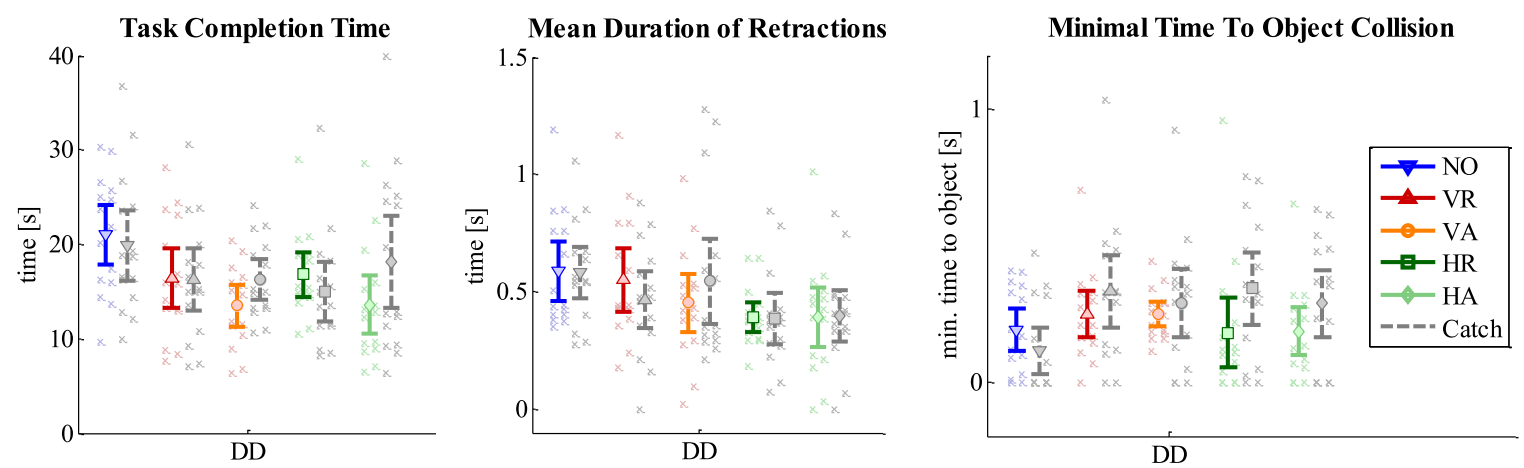

Fig. 6. Catch trial (CT) results of each experimental condition in the most difficult environment. The catch trials are shown in gray and the normal trials as previously reported in the represented colors. General figure representations are described in the caption of Fig. 4.

TABLE III

Percentage of Improvement Per SubJect for All Results

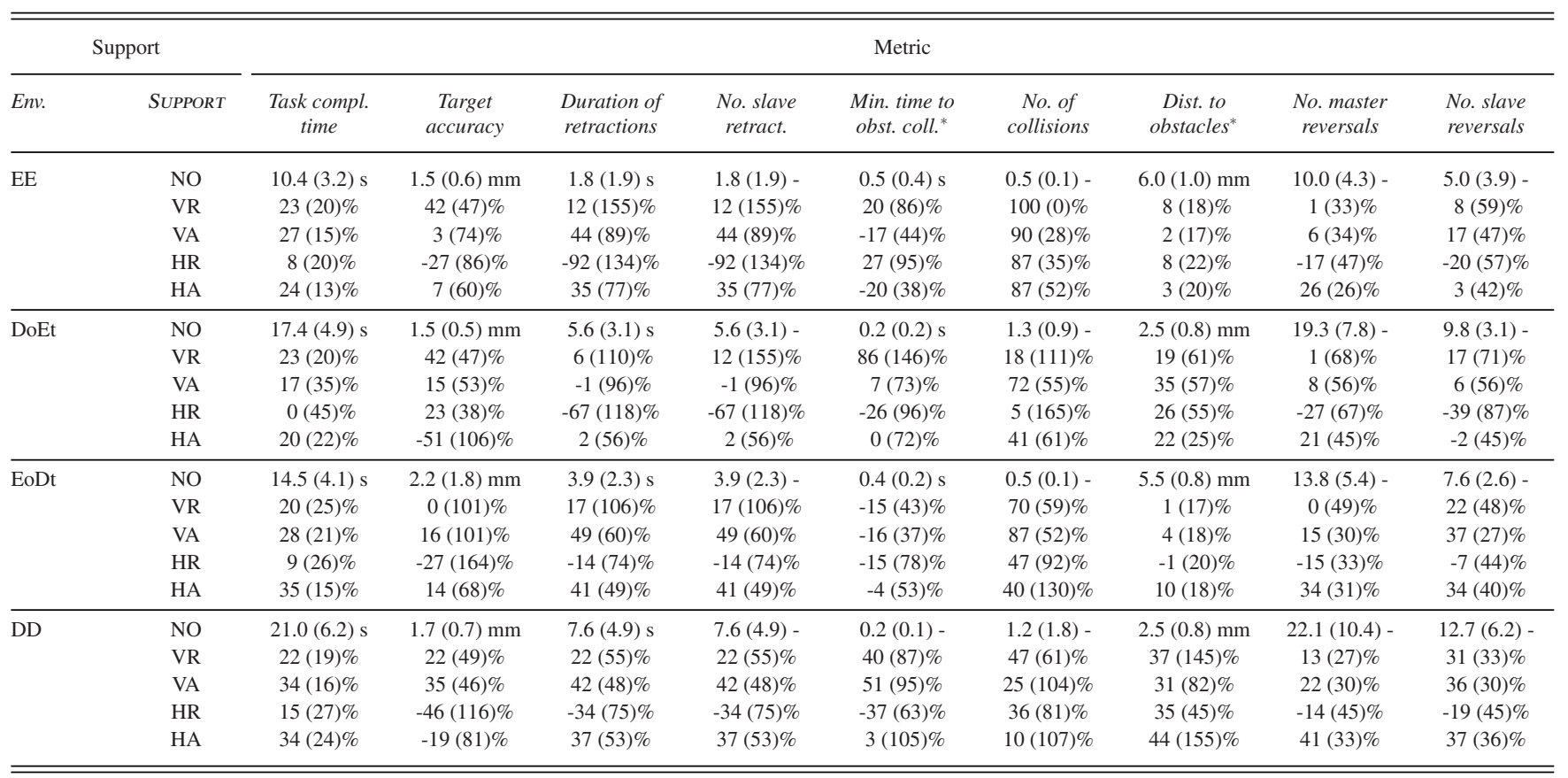

Displaying for the NO condition the mean results and standard deviation of the mean within brackets. The conditions with support are displayed as percentage of improvement per subject in each environment compared to the NO condition of that environment and the standard deviation of the mean in percentage within brackets.

${ }^{*}$ Improvements for these particular metrics are defined positive as increase compared to the NO condition, where other metrics are defined positive for a decrease.

environment than others. In general, it seems that all support systems are more effective in more difficult environments.

\section{Limitations, Cross-Checks, and Recommendations}

Unfortunately, due to the large amount of comparisons, our data were not sufficient to reveal subtle interactions between type of additional information and difficulty of the environment. The effect of support systems could, therefore, only be shown for all four environments as a whole.

Furthermore, the user interface used in this study limits the generalizability of the results. The user interface was used to create a challenging but easy to understand task, which would require the operator to rely on the given feedback to improve its performance. Therefore, this demonstrates clearly the difference between the methods, but not so much the size of the effect when applied at for instance car driving. The experimental order was counterbalanced and appropriate training was given prior to the experiment to all subjects. Nonetheless, learning effects were analyzed using a linear learning curve estimation for each subject, and the obtained parameters were averaged for all subjects. All learning rates were relatively small and had large variation. For example, task completion time had a mean reduction of $18 \%$ ( $\mathrm{SD}=21 \%$ ) over time (or trials), yet was based on inaccurate fits, $R^{2}<0.15$. The number of angular reversals had a reduction of $14 \%(\mathrm{SD}=33 \%)$ with $R^{2}<0.13$.

No evidence was found that any of the support systems resulted in overreliance or after effects [37]: Fig. 6 illustrates that the catch trials (implemented at the end of each experimental condition block) showed no significant difference compared with regular trials. 
Based on this study, it is recommended to offer both local environment information and global task information to the user either haptically or visually. This study shows when both types of information are offered with a support system (predicted vehicle dynamics combined with suggested path information), more improvements for conducting the task are found, provided that the user is not overloaded with information. Future work of operator modeling could explain the found effect of this human factors study. However, if not all of the information is available, even offering only the predicted vehicle dynamics visually substantially improves conducting the task. The latter could be used when only nearby environment information is available for steering a remote controlled vehicle.

\section{CONCLUSION}

In the context of steering a nonholonomic vehicle, a human factor experiment was conducted to determine the efficacy of four support system designs. The designs were based on different types of reflected information (predicted vehicle trajectory with or without suggested path) and through what modality this is conveyed (haptically or visually). For the experimental conditions studied, the following is concluded.

1) The support system designs that included both predicted vehicle trajectory and suggested path information improved task performance. No difference was found regarding the haptically or visually reflected information content.

2) Solely reflecting information of the predicted vehicle trajectory resulted in no improvements when offered haptically, but resulted in improved task performance and reduced control effort when offered visually.

3) Environments having more critical spatiotemporal constraints resulted in larger benefits (performance, safety, and effort) for all four support systems. No evidence was found that task criticality influenced the required type of additional information.

4) Additionally, catch trials did not provide evidence for overreliance of any support system.

These results indicate that offering additional information improves performance in a nonholomic steering task, especially when task criticality is high. When both types of information regarding the task are available (predicted vehicle trajectory with or without suggested path), it seems to be beneficial to reflect them both to the operator. The choice for presenting the additional information visually or haptically is not determined by this study and should be done in correspondence with the task and workload of the application it is used in.

\section{ACKNOWLEDGMENT}

This research resulted from a collaboration during the H-Haptics Autumn School, November 2013, organized by STW. The authors would like to thank R. J. Roesthuis for his help with setting up the experiment and analyzing the results.

\section{REFERENCES}

[1] J. G. W. Wildenbeest, D. A. Abbink, C. J. M. Heemskerk, F. C. T. Van Der Helm, and H. Boessenkool, "The impact of haptic feedback quality on the performance of teleoperated assembly tasks," IEEE Trans. Haptics, vol. 6, no. 2, pp. 242-252, Apr.-Jun. 2013.

[2] M. Massimino and T. Sheridan, "Variable force and visual feedback effects on teleoperator man/machine performance," in Proc. NASA Conf. Space Telerobot., 1989 pp. 89-98.

[3] W. S. Kim, F. Tendick, and L. W. Stark, "Visual enhancements in pickand-place tasks: Human operators controlling a simulated cylindrical manipulator," J. Robot. Autom., vol. 3, no. 5, pp. 418-425, 1987.

[4] C. P. Sayers, A. Lai, and R. P. Paul, "Visual imagery for subsea teleprogramming," in Proc. IEEE Int. Conf. Robot. Autom., 1995, vol. 2, pp. 1567-1572.

[5] P. M. T. Zaal, F. M. Nieuwenhuizen, M. M. van Paassen, and M. Mulder, "Modeling human control of self-motion direction with optic flow and vestibular motion," IEEE Trans. Syst. Man. Cybern. B, Cybern., vol. 43, no. 2, pp. 544-556, Sep. 2012.

[6] T. Sheridan, "Telerobotics," Automatica, vol. 25, no. 4, pp. 487-507, 1989.

[7] P. Milgram, A. Rastogi, and J. J. Grodski, "Telerobotic control using augmented reality," in Proc. 4th IEEE Int. Workshop Robot Human Commun., 1995, pp. 21-29.

[8] S. De Stigter, M. Mulder, and M. M. Van Paassen, "Design and evaluation of a haptic flight director," J. Guid. Control. Dyn., vol. 30, no. 1, pp. 35-46, Jan. 2007.

[9] J. Lüttgen and H. Heuer, "Robotic guidance benefits the learning of dynamic, but not of spatial movement characteristics," Exp. Brain Res., vol. 222, nos. 1/2, pp. 1-9, Oct. 2012.

[10] L. B. Rosenberg, "The use of virtual fixtures to enhance operator performance in time delayed teleoperation," J. Dyn. Syst. Control, vol. 49, pp. 29-36, 1993.

[11] A. Bettini and P. Marayong, "Vision-assisted control for manipulation using virtual fixtures," IEEE Trans. Robot., vol. 20, no. 6, pp. 953-966, Dec. 2004

[12] R. Prada and S. Payandeh, "On study of design and implementation of virtual fixtures," Virtual Real., vol. 13, pp. 117-129, 2009.

[13] T. M. Lam, M. Mulder, and M. M. Ren, "Haptic feedback for UAV teleoperation-Force offset and spring load modification," in Proc. IEEE Int. Conf. Syst., Man, Cybern., 2006, pp. 1618-1623.

[14] T. M. Lam, H. W. Boschloo, M. Mulder, and M. M. van Paassen, "Artificial force field for haptic feedback in UAV teleoperation," IEEE Trans. Syst. Man, Cybern. A, Syst. Humans, vol. 39, no. 6, pp. 1316-1330, Nov. 2009.

[15] N. Diolaiti and C. Melchiorri, "Tele-operation of a mobile robot through haptic feedback," in Proc. IEEE Int. Workshop Haptic Virtual Environ. Appl., Nov. 2002, pp. 17-18.

[16] C. P. Sayers and R. P. Paul, "An operator interface for teleprogramming employing synthetic fixtures," Presence (Camb)., vol. 3, no. 4, pp. 309-20, Jan. 1994.

[17] A. Majewicz and A. M. Okamura, "Cartesian and joint space teleoperation for nonholonomic steerable needles," in Proc. World Haptics Conf., 2013, pp. 395-400.

[18] P. Marayong and A. Okamura, "Speed-accuracy characteristics of humanmachine cooperative manipulation using virtual fixtures with variable admittance," Hum. Factor, J. Humans Factor Ergon. Soc., vol. 46, no. 3, pp. 518-532, 2004

[19] J. J. Abbott and A. M. Okamura, "Virtual fixture architectures for telemanipulation," in Proc IEEE Int. Conf. Robot. Autom., 2003, pp. 2798-2805.

[20] M. Steele and R. B. Gillespie, "Shared control between human and machine: Using a haptic steering wheel to aid in land vehicle guidance," in Proc. Hum. Factors Ergon. Soc. Annu. Meeting, 2001, pp. 1671-1675.

[21] D. Abbink, M. Mulder, and E. Boer, "Haptic shared control: smoothly shifting control authority?" Cogn. Technol. Work, vol. 14, pp. 19-28, 2012.

[22] D. A. Abbink and M. Mulder, "Exploring the dimensions of haptic feedback support in manual control," J. Comput. Inf. Sci., vol. 9, pp. 1-9, Mar. 2009.

[23] P. Griffiths and R. B. Gillespie, "Sharing control between human and automation using haptic interface: Primary and secondary task performance benefits," Human Factors, vol. 47, no. 3, pp. 574-590, 2005.

[24] R. J. Kuiper, J. C. L. Frumau, F. C. T. Van Der Helm, and D. A. Abbink, "Haptic support for Bi-manual control of a suspended grab for deep-sea excavation," in Proc. IEEE Int. Conf. Syst. Man, Cybern., Oct. 2013, pp. $1822-1827$. 
[25] P. Marayong and A. Okamura, "Speed-accuracy characteristics of humanmachine cooperative manipulation using virtual fixtures with variable admittance," Human Factors, vol. 46, pp. 518-532, 2004.

[26] H. Boessenkool, D. A. Abbink, C. J. M. Heemskerk, F. C. T. van der Helm, and J. G. W. Wildenbeest, "A task-specific analysis of the benefit of haptic shared control during telemanipulation," IEEE Trans. Haptics, vol. 6, no. 1, pp. 2-12, First Quarter 2013.

[27] B. A C. Forsyth and K. E. MacLean, "Predictive haptic guidance: Intelligent user assistance for the control of dynamic tasks," IEEE Trans. Vis. Comput. Graph., vol. 12, no. 1, pp. 103-113, Jan./Feb. 2006.

[28] T. Brandt, T. Sattel, and M. Bohm, "Combining haptic human-machine interaction with predictive path planning for lane-keeping and collision avoidance systems," in Proc IEEE Intell. Veh. Symp., 2007 pp. 582-587.

[29] W. Rankine, "On the dynamical principles of the motion of velocipedes," The Engineer, vol. 28, p. 79/129/153/175, 1869.

[30] A. V Christiansson, Hard Master, Soft Slave Haptic Teleoperation. Delft, Netherlands: Univ. Technol., 2007.

[31] R. G. D. Steel and J. H. Torrie, Principles and Procedures of Statistics: A Biometrical Approach. New York, NY, USA: McGraw-Hill, 1986.

[32] W. van Winsum, K. A. Brookhuis, and D. de Waard, "A comparison of different ways to approximate time-to-line crossing (TLC) during car driving," Accid. Anal. Prev., vol. 32, no. 1, pp. 47-56, Jan. 2000.

[33] S. G. Hart and L. E. Staveland, "Development of NASA-TLX (Task Load Index): Results of empirical and theoretical research," in Human Mental Workload. Amsterdam, The Netherlands: North Holland, 1988, pp. 239250.

[34] J. G. W. Wildenbeest, R. J. Kuiper, F. C. T. van der Helm, and D. A. Abbink, "Position control for slow dynamic systems: Haptic feedback makes system constraints tangible," in Proc. IEEE Int. Conf. Syst. Man, Cybern., Oct. 2014, pp. 3990-3995.

[35] M. Mulder, D. A. Abbink, and E. R. Boer, "Sharing control with haptics: Seamless driver support from manual to automatic control," Human Factors, vol. 54, pp. 786-798, May 2012.

[36] O. Khatib, "Real-time obstacle avoidance for manipulators and mobile robots," Int. J. Robot. Res., vol. 5, no. 1, pp. 90-98, Mar. 1986.

[37] J. C. F. de Winter and D. Dodou, "Preparing drivers for dangerous situations: A critical reflection on continuous shared control," in Proc. IEEE Int. Conf. Syst. Man, Cybern., Oct. 2011, pp. 1050-1056.

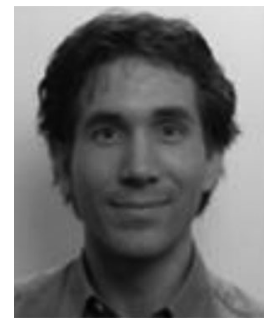

Roel J. Kuiper received the B.Sc. degree in mechanical engineering in 2006 and two M.Sc. degrees in mechanical engineering and offshore and dredging engineering in 2008 and 2012, respectively, all from the Delft University of Technology, Delft, The Netherlands, where he is currently working toward the Ph.D. degree.

His research interests include human-machine interfaces, teleoperation, rate control, shared control, haptic feedback, and haptic guiding systems.

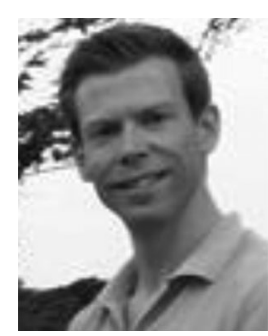

Dennis J. F. Heck received the B.Sc. and M.Sc. degrees in mechanical engineering in 2008 and 2011, respectively, and the Ph.D. degree in bilateral teleoperation.

His research interests include mechatronics, haptics, (tele)robotics, (non)linear dynamics, and (non)linear control.

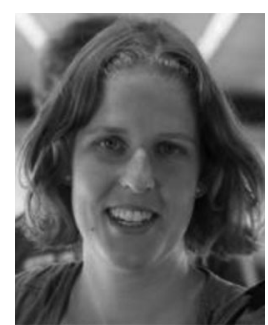

Irene A. Kuling received the B.Sc. degree in physics from Utrecht University, Utrecht, Netherlands, in 2008, and the M.Sc. degree in human-technology interaction from the Eindhoven University of Technology, Eindhoven, Netherlands, in 2011. She is currently working toward the $\mathrm{Ph} . \mathrm{D}$. degree in visuohaptic perception with Vrije Universiteit Amsterdam, Amsterdam, Netherlands.

Her research interests include human perception, haptics, and psychophysics.

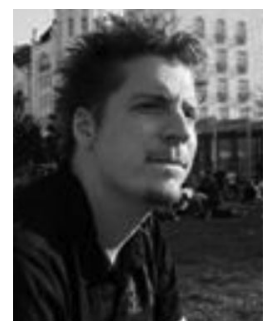

David A. Abbink (M'12-SM'14) received the M.Sc. and $\mathrm{Ph} . \mathrm{D}$. degrees in mechanical engineering from the Delft University of Technology, Delft, Netherlands, in 2002 and 2006, respectively.

$\mathrm{He}$ is currently an Assistant Professor with the Delft Haptics Laboratory, Delft University of Technology. His research interests include haptics, driver support systems, shared control, system identification, and neuromuscular analysis. 